\title{
SALINIDAD Y NUTRIENTES EN LAS LAGUNAS COSTERAS DEL DELTA DEL EBRO
}

\author{
F.A. Comín; M. Menéndez; E. Forés \\ Departamento de Ecología, Facultad de Biología, Universidad de Barcelona. Diagonal 645. Barcelona 08028
}

Palabras Clave: Coastal lagoons, Physical and Chemical Limnology, Ebro Delta (Spain).

\section{ABSTRACT \\ SALINITY AND NUTRIENTS IN THE EBRO DELTA COASTAL LAGOONS}

\begin{abstract}
Seasonal changes of salinity, water level and nutnents were studied in coastal lagoons of the Ebro Delta. The data presented in this paper show the differences between these lagoons and others around the world.

The freshwater from the nver and ricefields flows into the lagoons from Apnl to December. This is the cause of the low salinity values and the relatively high water lavels observed during this penod of the year in contrast to the values from January to Apnl. The intensity of these fluctuations depends on the flow rates.

The lagoons are in an advanced process of eutrophication. The hydrological cycle patially controls the seasonal changes of phosphorus and nitrogen and two relative maxima are observed in all the lagoons. Those of phosphorus occured in March and October and those of nitrogen (dissolved inorganic oxydized nitrogen) in winter and spnng. These pattems of vanation are discussed for the different lagoons.
\end{abstract}

Buda lagoon data are exceptions to the general model of hydrological cycle and the nutnent concentrations are lower.

\section{INTRODUCCION}

Las lagunas costeras, son ecosistemas muy fluctuantes caracterizados fundamentalmente por la mezcla de aguas continentales y marinas. La intensidad y frecuencia de esta mezcla, determina en su mayor parte los cambios estacionales de las características químicas del agua y de las poblaciones biológicas que las habitan (Ketchum, 1954; Postma, 1969; Margalef, 1969).

Las lagunas costeras del Delta del Ebro, reciben mayoritariamente el agua dulce por canales que provienen del río, que además recogen el agua utilizada en campos de arroz. Las necesidades del cultivo del arroz, imponen un régimen hidrológico que se caracteriza por entradas de agua durante el periodo de cultivo (mayo-noviembre) y, cese de estos aportes y consiguiente intercambio de agua con el mar durante el resto del año (diciembre-abril) (Comín \& Ferrer, 1978).

Este modelo simplificado, presenta variaciones de una laguna a otra (Comin, 1984) que explican diferencias en los cambios estacionales de las comunida-

Limnética 3: 1-8 (1987)

(C) Asociación Española de Limnología, Madrid. Spain des y de las características químicas (Comin, 1982). En este artículo se presentan las diferencias entre las distintas lagunas costeras del Delta del Ebro, con información referente a los cambios de salinidad y elementos nutritivos.

\section{MATERIAL Y METODOS}

Se recogieron muestras de agua $($ a $20 \mathrm{~cm}$. de profundidad) desde enero de 1983 a marzo de 1984 en las localidades señaladas en la figura 1. Muestras no filtradas y fijadas con cloroformo inmediatamente después de recolectadas se conservaron a $4^{\circ} \mathrm{C}$ hasta el momento de realizar los análisis de elementos nutritivos. Los métodos utilizados son los detallados en Margalef $\boldsymbol{e t}$ al. (1976). «In situ», se midieron la temperatura, $\mathrm{pH}$, conductividad y nivel de agua.

\section{RESULTADOS}

Las lagunas costeras del Delta del Ebro son muy 


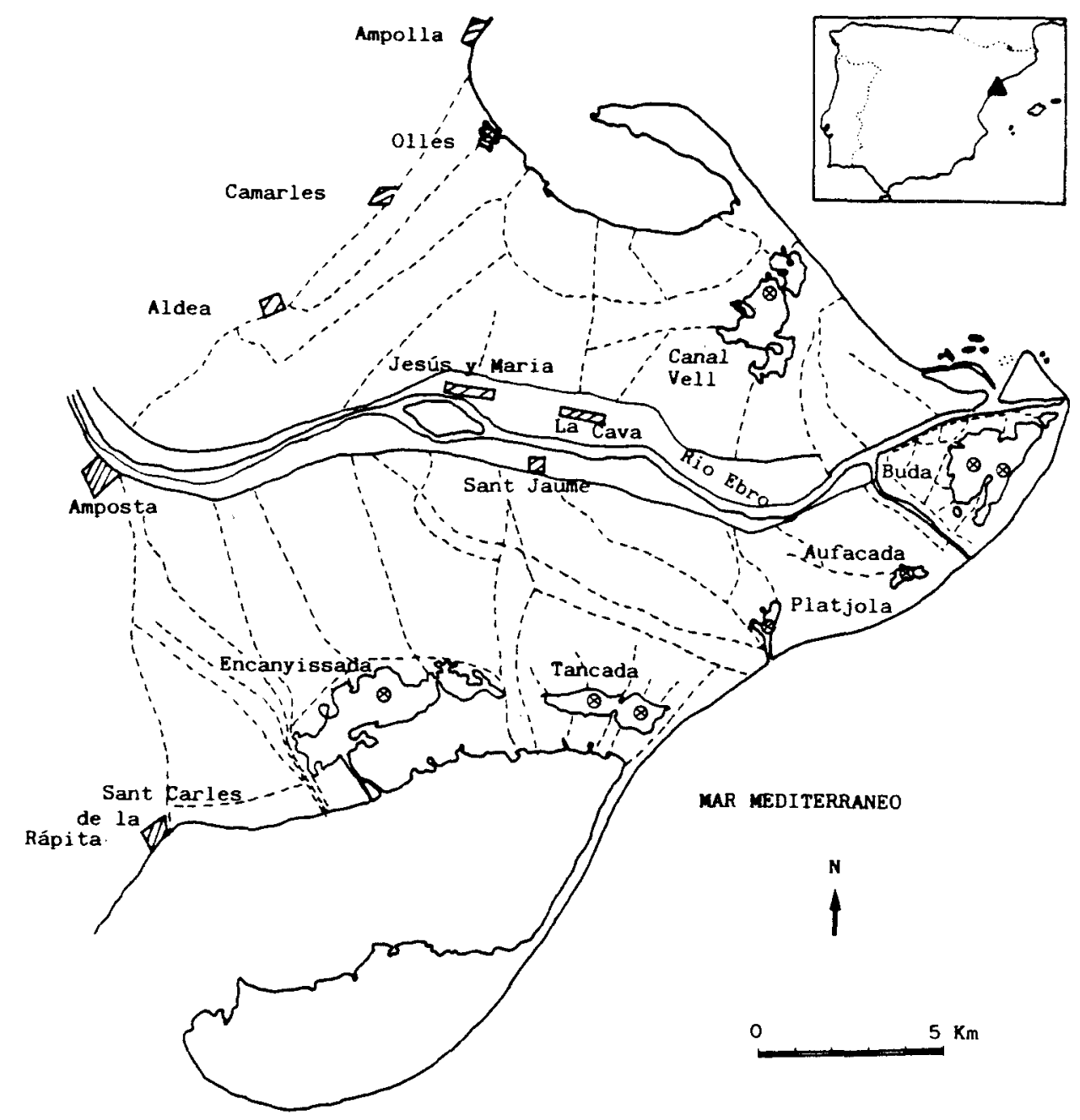

Figura 1.- Area de estudio. ( ${ }^{\circ}$ Estaciones de muestreo).

Study area ( ${ }^{\circ}$ sampling sites).

someras. Su profundidad media es inferior a $50 \mathrm{~cm}$ (Comín, 1984). Sólo la Encanyissada mantiene durante varios meses un nivel de agua superior a $1 \mathrm{~m}$. (fig. 2).

El nivel de agua es más alto durante los meses de menor salinidad, que corresponde en general a los meses de mayo a diciembre (fig. 3). Durante el otro periodo del año, de enero a abril, presentan valores de conductividad relativamente altos y niveles de agua bajos (tabla 1). Excepciones a este modelo simplificado son la Alfacada, con un valor máximo de conductividad en el mes de mayo, y Buda, donde el valor máximo se observó en agosto.

En la mayoría de las lagunas el pH permanece más alto y dentro de un rango de variación más estrecho durante los meses de enero a abril que durante el pe- riodo de mayo a diciembre, coincidiendo con unos valores de alcalinidad más elevados (tabla 1). El coeficiente de correlación entre ambos parámetros se encuentra entre 0.60 (Platjola) y 0.73 (Buda).

Comin (1984) comprobó que el pH y la alcalinidad del agua de las lagunas varía en relación directa con los del agua que llega por los canales de riego durante el periodo de mayo a diciembre, mientras que durante los meses de invierno amortiguan sus variaciones debido a que la entrada de agua de mar a las lagunas tienen un efecto tamponador de ambos parámetros. Se ha comprobado que existe una relación importante entre el $\mathrm{pH}$ y la alcalinidad del agua de las lagunas y la de los canales y también el efecto tamponador del agua de mar al entrar en las lagunas (Comín, 1984). 


\begin{tabular}{|c|c|c|c|c|c|c|c|c|}
\hline & & EN & TA & PI & $\mathrm{AL}$ & $\mathrm{BU}$ & $\mathrm{CV}$ & OLL \\
\hline $\begin{array}{l}\text { Nivel del } \\
\text { agua }(\mathrm{cm})\end{array}$ & $\begin{array}{l}M \\
1 \\
D \\
E \\
1 \\
A\end{array}$ & $\begin{array}{c}102-130 \\
71-97\end{array}$ & $\begin{array}{l}54-85 \\
30-66\end{array}$ & $\begin{array}{r}110-120 \\
15-104\end{array}$ & $\begin{array}{l}37-70 \\
17-42\end{array}$ & $\begin{array}{l}66-90 \\
51-68\end{array}$ & $\begin{array}{l}46-77 \\
30-49\end{array}$ & $\begin{array}{l}29-55 \\
25-33\end{array}$ \\
\hline $\begin{array}{l}\text { Conductivi- } \\
\text { dad. } \mathrm{mS} / \mathrm{cm}\end{array}$ & $\begin{array}{l}M \\
1 \\
D \\
E \\
A\end{array}$ & $\begin{array}{l}4-10 \\
10-40\end{array}$ & $\begin{array}{r}8-20 \\
17-45\end{array}$ & $\begin{array}{l}2-18 \\
11-39\end{array}$ & $\begin{array}{c}3-33 \\
10-29\end{array}$ & $\begin{array}{r}3-25 \\
11-18\end{array}$ & $\begin{array}{r}3-31 \\
14-47\end{array}$ & $\begin{array}{c}1-20 \\
16-27\end{array}$ \\
\hline $\mathrm{pH}$ & $\begin{array}{l}M \\
\dot{D} \\
D \\
E \\
d\end{array}$ & $\begin{array}{l}7.9-8.4 \\
7.2-8.1\end{array}$ & $\begin{array}{l}8-9.6 \\
8-8.5\end{array}$ & $\begin{array}{l}7.6-8.3 \\
7.9-8.2\end{array}$ & $\begin{array}{l}7.5-8.47 \\
7.7-8.38\end{array}$ & $\begin{array}{l}7.5-8.9 \\
8.3-8.8\end{array}$ & $\begin{array}{l}7.6-8.2 \\
7.8-8.3\end{array}$ & $\begin{array}{l}7.6-8.4 \\
8.1-8.4\end{array}$ \\
\hline $\begin{array}{l}\text { Alcalinidad } \\
\text { meq/1 }\end{array}$ & $\begin{array}{l}M \\
\dot{D} \\
E \\
\hat{A}\end{array}$ & $\begin{array}{l}2.5-4.6 \\
3.8-4.4\end{array}$ & $\begin{array}{l}2.6-5 \\
4.6-4.8\end{array}$ & $\begin{array}{l}3-7 \\
4-9.8\end{array}$ & $\begin{array}{c}3.6-5.5 \\
3-5.8\end{array}$ & $\begin{array}{l}2.1-4.9 \\
3.2-4.9\end{array}$ & $\begin{array}{l}3.5-4.4 \\
1.9-4.4\end{array}$ & $\begin{array}{c}3-5.1 \\
2.6-5.2\end{array}$ \\
\hline $\begin{array}{l}\text { rosfatos } \\
(\mu g-a t / 1)\end{array}$ & $\begin{array}{l}M \\
1 \\
D \\
E \\
A\end{array}$ & $\begin{array}{l}0.2-18 \\
0.3-4.7\end{array}$ & $\begin{array}{l}0.6-5.7 \\
0.9-9\end{array}$ & $\begin{array}{l}0.5-22 \\
0.6-18\end{array}$ & $\begin{array}{c}0.2-9.6 \\
0.4\end{array}$ & $\begin{array}{l}0.3-1.8 \\
0.4-0.5\end{array}$ & $\begin{array}{l}0.1-8.5 \\
0.3-5.9\end{array}$ & $\begin{array}{l}0.2-60.6 \\
0.7-10.9\end{array}$ \\
\hline $\begin{array}{l}\text { Nitratos } \\
(\mu g-a t / 1)\end{array}$ & $\begin{array}{l}M \\
\vdots \\
E \\
A\end{array}$ & $\begin{array}{l}0-51 \\
6-28\end{array}$ & $\begin{array}{l}0-11.3 \\
0.2-28\end{array}$ & $\begin{array}{l}0-17.7 \\
5.5-42.7\end{array}$ & $\begin{array}{c}0-90 \\
0.4-21.8\end{array}$ & $\begin{array}{c}0-9.5 \\
82.6-40\end{array}$ & $\begin{array}{l}0-42 \\
2-100\end{array}$ & $\begin{array}{l}10-138 \\
0-68.4\end{array}$ \\
\hline $\begin{array}{l}\text { Nitritos } \\
(\mu g-a t / 1)\end{array}$ & $\begin{array}{l}M \\
d \\
E \\
A\end{array}$ & $\begin{array}{l}0.3-9.5 \\
0.3-3\end{array}$ & $\begin{array}{l}0-4.1 \\
0.4-3.1\end{array}$ & $\begin{array}{l}0-2.9 \\
0.4-6.8\end{array}$ & $\begin{array}{l}0-1.5 \\
0.2-2.8\end{array}$ & $\begin{array}{c}0.6-2.1 \\
0-2.9\end{array}$ & $\begin{array}{l}0-11.5 \\
0.3-5.7\end{array}$ & $\begin{array}{c}0.2-76 \\
0-13\end{array}$ \\
\hline
\end{tabular}

Tabla 1.- Rangos de variación de algunos parámetros en las lagunas costeras del Delta del Ebro en dos periodos del año (mayodiciembre,enero-abril).

Ranges of vanation of several parameters in the Ebro Delta coastal lagoons dunng two penods ofthe year (May-December,January-April).

En las concentraciones de elementos nutritivos no se observan diferencias claras en los dos periodos del año (tabla t). Respecto al fósforohay una clara dife. rencia entre las lagunas Encanyissada, Platjola y Olles y el resto (fig. 4). En las tres citadas la mayoría de los valores son superiores a 1 pg-at PRS/1. y los máximos son superiores a $15 \mu \mathrm{g}$-at PRS/1. En el resto de lagunas la concentración es inferior a $1 \mu \mathrm{g}$-at/l. durante una parte notable del año y los valores máximos no sobrepasan $10 \mu \mathrm{g}$-at/l. Las concentraciones más bajas corresponden a la laguna de Buda con valores no superiores a $2.5 \mu \mathrm{g}$-at PRS $/ 1$.

En todas las lagunas se detectó un pico en el mes de octubre y otro al final de invierno o principios de primavera. En la Encanyissada, Platjola, Olles y Canal Vell, el primero es el máximo anual. En Alfacada y Tancada el máximo anual es el de invierno. También en Buda (Comin, 1984).

Respecto al nitrógeno disuelto en forma inorgánica oxidada tampoco existen diferencias de concentración relacionadas con los periodos señalados a propósito de la salinidad. Las mayores concentraciones a lo lar- go del año correspondieron a las lagunas Encanyissada, Platjola y Olles (fig. 5). La máxima concentración absoluta fue de 143 pg-at $N-(N O ;+N O ;) / 1$; en Olles. La mayoría de los valores quedaron entre 10 y $50 \mu \mathrm{g}-\mathrm{at} / \mathrm{l}$, en estas tres lagunas. En las demás la mayoria de datos quedaron dentro del rango 0-10 $\mu \mathrm{g}$ at/l. En Alfacada y Canal Vell se observaron máximos anuales de 90 y $100 \mu \mathrm{g}$-at $/ \mathrm{l}$., respectivamente. En Tancada y Buda fueron de 30 y $40 \mu \mathrm{g}$-at/1, ambos en marzo.

Datos previos de nitrogeno inorgánico disuelto en forma de amonio muestran variaciones anuales inversas a las de las formas oxidadas (Comin, 1984). En la Encanyissada los máximos son en junio y julio (70 pg-at $\mathrm{N}-\mathrm{NH}_{+}^{+} / 1$.) y en la Tancada en los mismos meses (18 pg-at $N$-NH $\$ /[$ ).

\section{DISCUSION}

Los datos presentados demuestran que el régimen hidrológico de las lagunas costeras del Delta del Ebro, 
NIVEL DEL AGUA

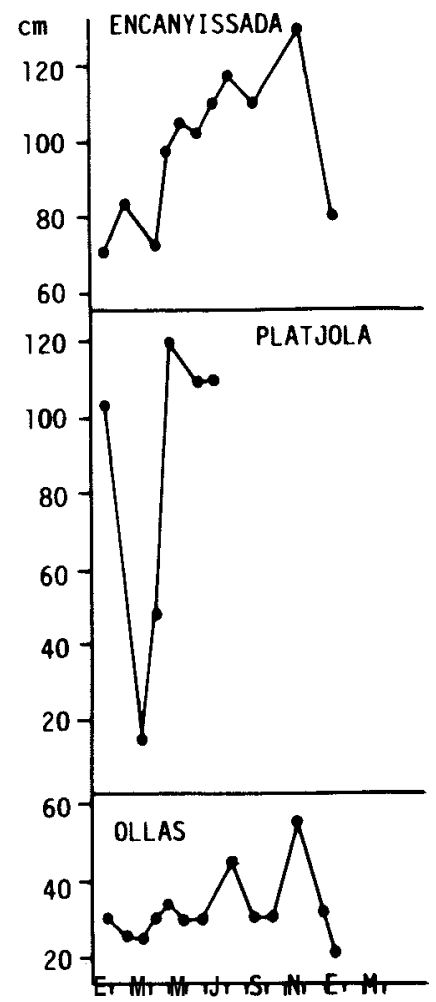

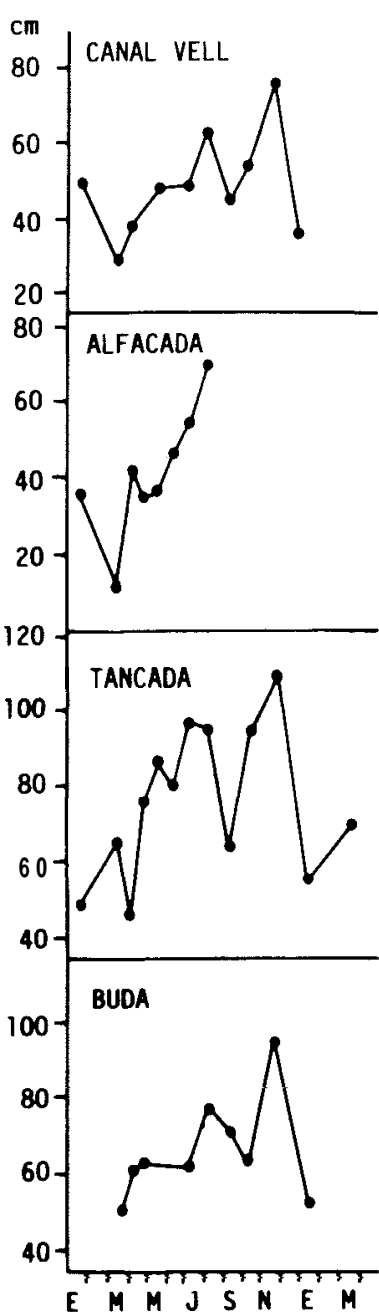

Figura 2.- Variación estacional del nivel del agua en las lagunas costeras del Delta del Ebro. Seasonal changes ofthe water level in the Ebro Delta coastal lagoons.

está determinado por los aportes de agua dulce provenientes de canales artificiales. El modelo general de variación estacional de la salinidad, es inverso al de la mayoría de las lagunas costeras del Mediterráneo, en las que los valores más altos de salinidad se observan en verano (mínimo de lluvias y máxima evaporación) y los más bajos en invierno (Nisbet et al., 1958; Colombo, 1973).

El equilibrio del nivel de agua entre las lagunas y el mar está desplazado a favor de las primeras cuando tienen lugar los aportes de agua dulce. Así se manifiesta, en general, una relación inversa entre el nivel y la salinidad del agua (fig. 6). En algunas lagunas esta relación es mayor (Encanyissada, coef. de correl. = -0.84 y Platjola coef. de correl. $=-0.86)$ que en otras ( Tancada coef. de correl. $=-0.43$ y Olles coef. de co- rrel. $=0.41)$. La laguna de Buda, constituye una excepción ya que el agua se intercambia principalmente con la desembocadura del río, donde el agua dulce y marina, se mezclan según una circulación estuárica positiva (Comín, 1984), de manera que el agua que entra en la laguna tiene una salinidad intermedia entre ambas.

Las diferencias entre lagunas, se ponen de manifiesto si se compara la fluctuación de la salinidad con la salinidad media. En la fig. 7 se han representado en un sistema de ejes de coordenadas las posiciones relativas de las lagunas. El eje de abscisas queda definido por el porcentaje de la salinidad respecto a la salinidad máxima observada en todas las lagunas y el eje de ordenadas por el porcentaje de la suma de las variaciones (sin signo) de la salinidad respecto a la fluc- 


\section{CONDUCTIVIDAD}

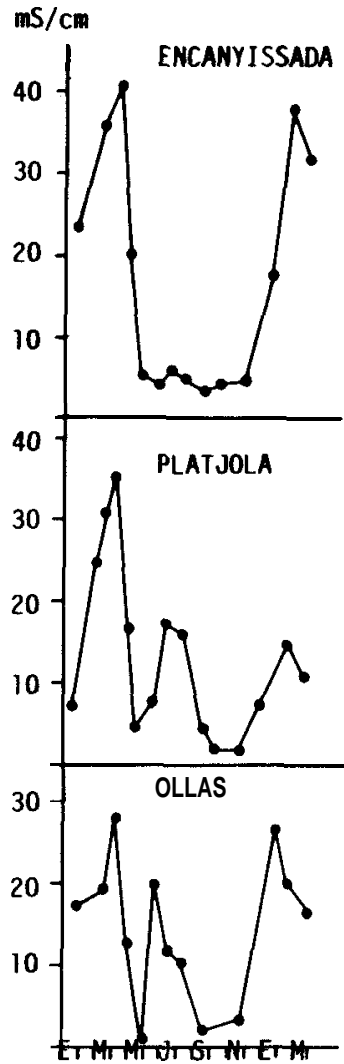

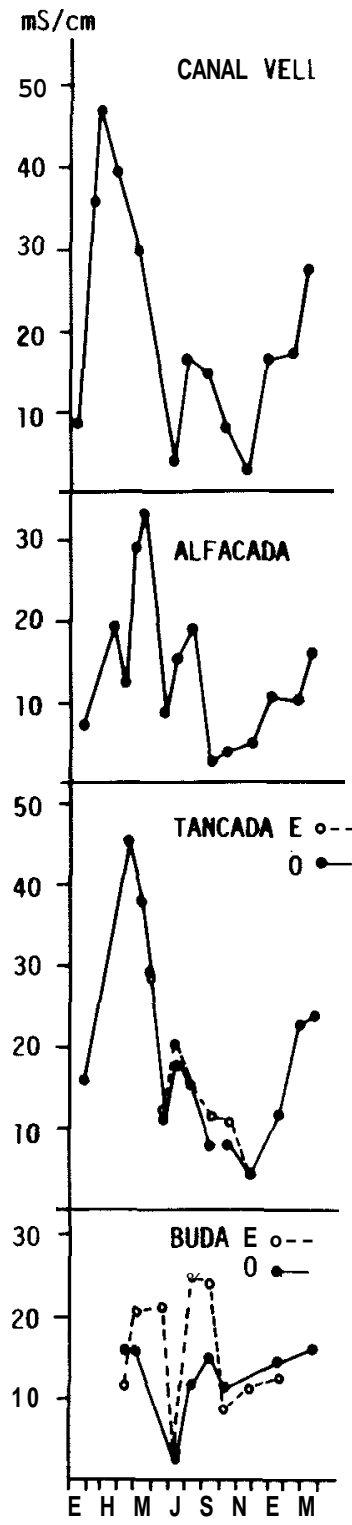

Figura 3.- Ciclo anual de la conductividad. Annual cycle of the conductivity.

tuación máxima entre todas las lagunas. Este esquema tiene validez para un conjunto de lagunas costeras e incluso para otros tipos de ecosistemas y puede servir para justificar diferencias en la persistencia de las poblaciones biológicas en distintas lagunas. En este esquema, los ecosistemas marinos quedarían situados en el extremo inferior derecho (salinidad elevada y constante). El sector superior derecho correspondería a lagunas costeras con una renovación elevada de agua y dominadas por el intercambio con el mar. En el sector inferior izquierdo se ubicarían lagunas con predominancia de agua dulce y pocas variaciones estacionales de la salinidad, en contraste con las del Delta del Ebro, en las que el agua dulce es predominante y los cambios de salinidad frecuentes y grandes durante el año (zona superior izquierda).

Los datos presentados sobre las variaciones estacionales de las concentraciones de elementos nutritivos, ofrecen peculiaridades en parte relacionables con el régimen hidrológico ya explicado. El rango de las 

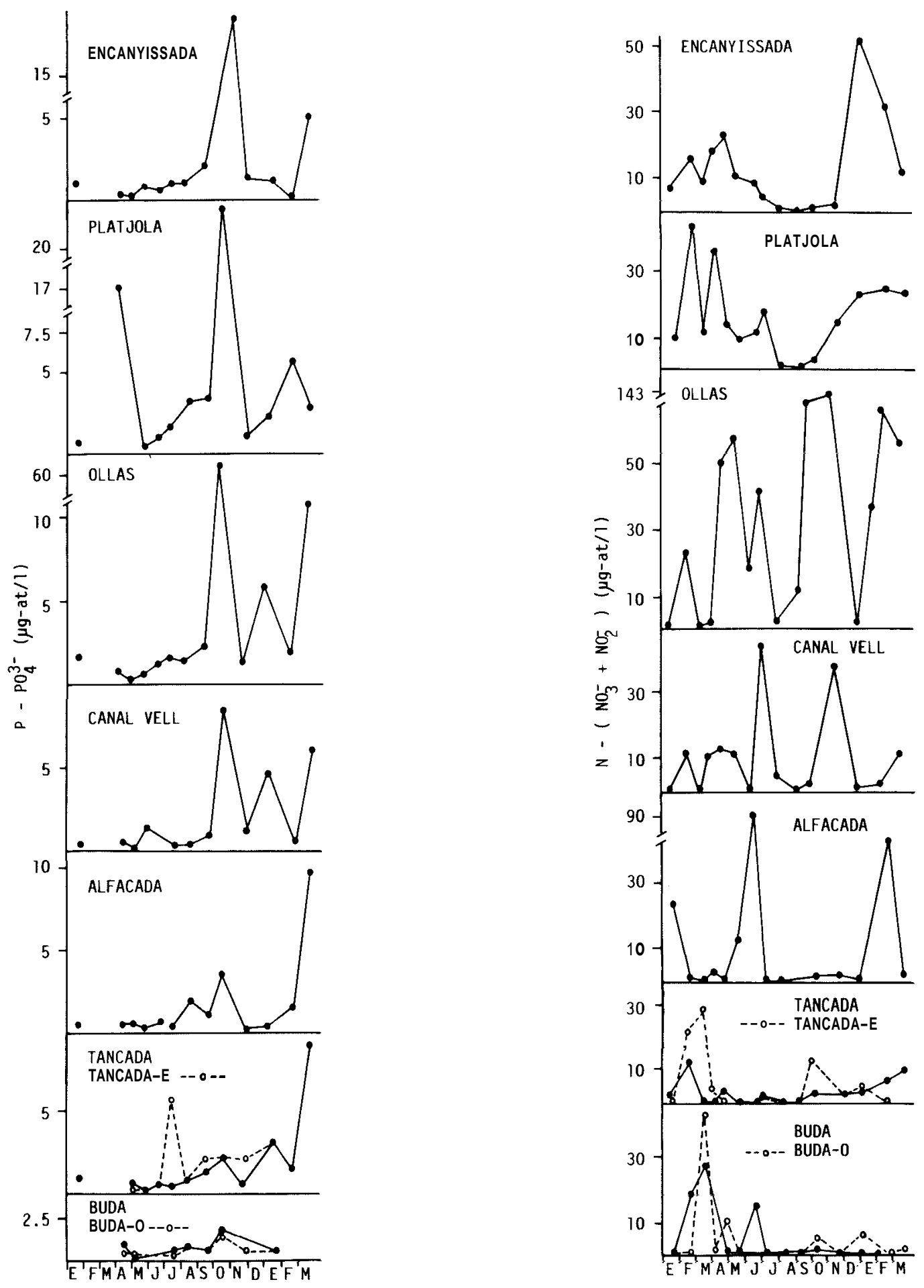

Figura 4.- Variación estaciona] de la concentración de fósforo reactivo soluble (pg-at $\mathrm{P}-\mathrm{PO} \equiv / 1)$.

Seasonal changes of phosphate ( $\mu \mathrm{g}$-at PRS/1).

Figura 5.- Ciclo anual de la concentración de nitrógeno $\left(\mathrm{pg}-\right.$ at $\left.\mathrm{N}-\left(\mathrm{NO}_{3}^{-}+\mathrm{NO}_{2}^{-}\right) / \mathrm{l}\right)$.

Annual cycle ofnitrate plus nitnte $\left(\mu \mathrm{g}\right.$-at $\left.\mathrm{N}-\left(\mathrm{NO}_{3}^{-}+\mathrm{NO}_{2}^{-}\right) / \mathrm{l}\right)$. 

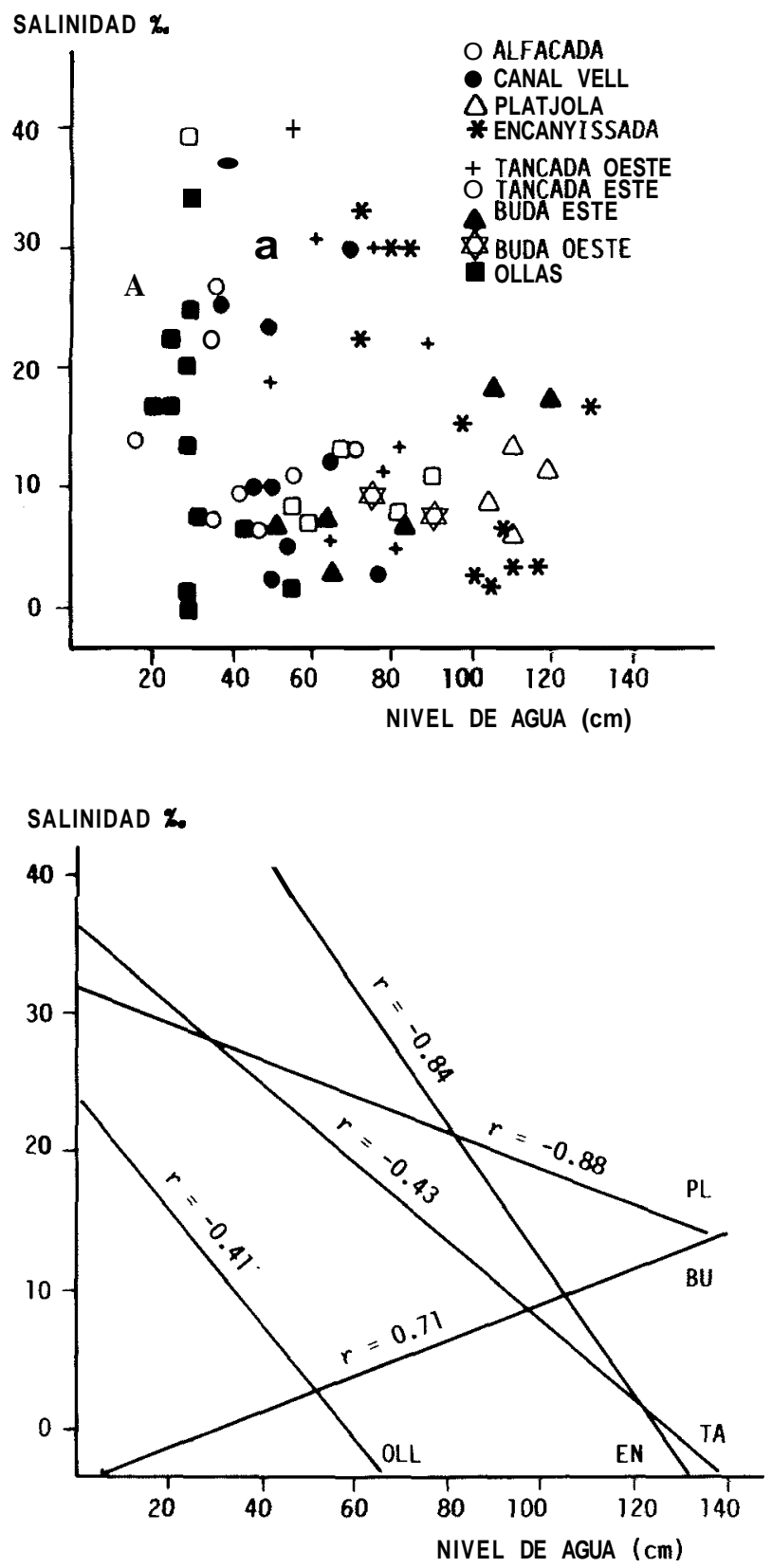

Figura 6.- Relación entre el nivel de agua y la salinidad. Se han dibujado las rectas de regresión para las lagunas con coeficientes de correlación significativos (p0.05).

Relationship between salinity and water level. Only significant regression lines have been drawn ( $\mathrm{p} 0.05)$.

concentraciones de fosfatos, es de 0.1 a 100 pg-at PRS $/ 1$., aunque la mayoría de los valores quedan entre 0.1 y $20 \mathrm{pg}$-at PRS/1. Son superiores al rango de

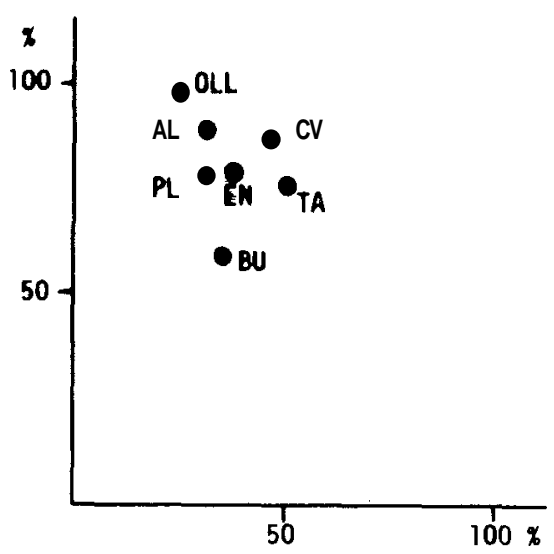

Figura 7.- Representación de la posición relativa que ocupan las lagunas costeras del Delta del Ebro en un espacio ideal definido por la media y la fluctuación anual de la salinidad (véase el texto).

The relationship between fluctuations and mean salinity as percentage of the maximum fluctuation and mean salinity (see the text).

0.1 a $5 \mu \mathrm{g}$-at $/ 1$. citados por Nixon (1982) para lagunas costeras del Mediterráneo y otros mares. Indican un grado de eutrofización avanzado que es proporcional a los aportes de agua dulce por los canales. Las lagunas con mayor concentración de fosfato son las que reciben también una mayor descarga de agua dulce (Encanyissada, Platjola, Olles). La laguna Buda, que tiene un area de drenaje artificial mínima, es la única que mantiene una concentración de fosfatos inferior a $2.5 \mu \mathrm{g}-a t / 1$. durante todo el año. La variación estacional sigue un patrón similar en todas las lagunas. El máximo del mes de octubre coincide con la descarga de agua procedente del vaciado de los campos de arroz después de la cosecha y es más alto en las lagunas que reciben mayores aportes. El otro máximo ocurre en invierno. Puede ser causado por la mineralización de la materia orgánica acumulada durante el resto del año, pero no por un menor consumo por el fitoplancton ya que su actividad en ese periodo es mayor (Comin, 1984). Este proceso sería análogo para el nitrógeno ya que se observa un máximo de este elemento en todas las lagunas durante el invierno.

El rango de variación de la mayoría de las concentraciones de nitrógeno (0-50 pg-at $\mathrm{N}-\left(\mathrm{NO}_{3}^{-}+\right.$ $\left.\mathrm{NO}_{-}^{-}\right) / 1$.) es más amplio que el de fósforo. Esta diferencia puede atribuirse a que el nitrógeno es captado y liberado en actividades biológicas en mayor cantidad que el fósforo (Nixon, 1982). Las lagunas Encanyissada, Platjola y Olles, tienen las concentraciones mas altas en abril y mayo. Se deben a los vertidos de 
agua dulce correspondientes al inicio del cultivo de arroz. Esto se confirma al observar que estos máximos en Alfacada y Canal Vell están retrasados a junio y julio, respectivamente, cuando la conductividad es mínima.

Es común a todas las lagunas una baja o inapreciable concentración de nitrógeno inorgánico oxidado durante los meses cálidos. Esto es un hecho que ocurre en numerosos ambientes acuáticos costeros, puede estar relacionado con el consumo por el fitoplancton junto a los procesos de desnitrificación (Valiela, 1984).

Teniendo en cuenta solamente estos datos, la relación N:P es en general inferior a 16:1 durante los meses de verano. Sin embargo si se incluyen los datos de las concentraciones de amonio, durante el periodo de mayo a diciembre son siempre superiores al cociente 16:1 mientras que durante los meses de enero a abril hay periodos con valores inferiores. Según esto el control del crecimiento de las poblaciones algales correspondería al fósforo durante los meses de descarga de agua dulce y al nitrógeno durante los meses de salinidad elevada, que es la situación más generalizada en cuanto a limitación de nutrientes en aguas dulces y marinas.

\section{BIBLIOGRAFIA}

Colombo, O.C. 1973. «Lagoons». En Barnes, R.S.K. (ed.) The coastline. John Wiley \& Sons Ltd.-: 63-82.
Comín, F.A. \& Ferrer, X., 1978. Les llacunes litorals. Quad. Ecol. Apl. 4: 51-68.

Comín, F.A. 1982. Seasonal change of phytoplankton in three coastal lagoons of the Ebro Delta in relation to the environmental factors. En P. Lasserre \& H. Postma (ed.) Coastal lagoons Oceanol. Acta 4:259-267.

Comín, F.A. 1984. Características físicas y químicas y fitoplancton de las lagunas costeras, Encañizada, Tancada y Buda (Delta del Ebro), Oecol aquatica, 7: 79-162.

Ketchum, B.H. 1954. Relation between circulation and planktonic populations in estuaries. Ecology 35: 191-200.

Margalef, R. 1969. Comunidades planctónicas en lagunas litorales. En Ayala-Castañares \& Phleger (ed.) Lagunas costeras. Mem. Simp. Intern. Lagunas costeras. UNAMUNESCO. México D.F.: 545-562.

Margalef, R.; Planas, D.; Armengol, J.; Vidal, A.; Prat, N.; Guiset, A.; Toja, J.; Estrada, M., 1976. Limnología de los embalses españoles. Dirección General de Obras Hidráulicas. M. ${ }^{\circ}$ de Obras Públicas, publ. n. ${ }^{\circ} 123$ (422 pp.).

Nisbet, M.; Petit, G.; Schachter, D., 1958. Caracteres chimiques de quelques ètangs Mediterraneens classification des eaux saumâtres Verh. internat. Ver. Limnol. XIII: 672-675.

Nixon, S.W., 1982. Nutrients dynamics, primary production and fisheries yields of lagoons. Oceanol. Acta n.$^{\circ}$ sp. Actes Symposium lntemational sur les lagunes côtières. SCOR/ IABO/UNESCO, Bordeaux: 357-371.

Postma, H. 1969. Sediment, transport and sedimentation in the marine environment. en Lauff, G.H. Estuaries. AAAS n. 83: 158-179.

Valiela, I., 1984. Marine Ecological Processes. SpringerVerlag. New York, Inc. (564 pp.). 